\title{
Construcción y caracterización de un destilador solar de una vertiente con superficie reflectora
}

\author{
Construction and characterization of a single slope solar still with reflecting \\ surface
}

${ }^{1}$ Carlos Polo Bravo
${ }^{2}$ Adolfo Pérez Cruz

ORCID: 0000-0001-9491-2323

\begin{abstract}
RESUMEN
El presente trabajo tuvo como objetivo construir y caracterizar un destilador solar de una vertiente (DSV) para desalar agua de mar con superficie reflectora, bajo las condiciones meteorológicas de la ciudad de Tacna, utilizando la energía solar; en la construcción se utilizaron materiales locales. Las dimensiones internas del destilador son de $80 \mathrm{~cm}$ de altura en la parte trasera, $35 \mathrm{~cm}$ de altura en la parte frontal, $75 \mathrm{~cm}$ de ancho, 135 $\mathrm{cm}$ de largo, cámara de volumen de $0,58 \mathrm{~m}^{3}$, laterales y fondo de $3 \mathrm{~cm}$ de espesor, el cobertor tiene una inclinación de $30^{\circ}$ y área de $1,35 \mathrm{~m}$ x $0,8 \mathrm{~m}\left(1,08 \mathrm{~m}^{2}\right)$. Se colocaron depósitos para colectar el agua destilada en las cuatro paredes del sistema, un espejo en la parte trasera como reflectante y sensores de temperatura para la caracterización térmica. Durante un día con un volumen de 25 litros de agua salada en la cubeta se obtuvo 2,19 litros de agua destilada, en el cobertor de vidrio 1,12 litros (51,12 \%), pared lateral izquierda 0,52 litros de $(23,75 \%)$ y 0,55 litros de la pared lateral derecha $(25,23 \%)$, la pared reflectante aislada no condensó vapor de agua; la concentración de sal del agua de mar de un valor inicial de 41,3 bajó a 0,004 gramos de sal/litro del agua, en tanto que el pH de 7,87 y 7,03. El rendimiento de destilación en las paredes verticales laterales de vidrio fue de $3,05 \mathrm{l} / \mathrm{m}^{2}$ día, mayor que el cobertor inclinado $30^{\circ}$ de vidrio igual a 0,75 $1 / \mathrm{m}^{2}$ día, aproximadamente en cuatro veces respecto al cobertor inclinado en $30^{\circ}$. Los resultados indicaron que el DSV es una buena alternativa para destilar agua de mar a pequeños volúmenes por día, a bajo costo para consumo humano y otros usos, en el caso de requerir mayores volúmenes de agua destilada se pueden usar los sistemas en forma modular.
\end{abstract}

Palabras clave: Agua, destilador, solar, superficie reflejante, temperatura, volumen.

\begin{abstract}
The aim of the research was to build and characterize a single-slope solar still (DSV) to desalinate seawater with a reflecting surface, under the meteorological conditions of the city of Tacna, using solar energy; local materials have been used in the construction. The internal dimensions of the still are $80 \mathrm{~cm}$ high at the rear, $35 \mathrm{~cm}$ high at the front, $75 \mathrm{~cm}$ wide, $135 \mathrm{~cm}$ long, $0.58 \mathrm{~m} 3$ chamber volume, sides and bottom $3 \mathrm{~cm}$ thick, the cover has an inclination of $30^{\circ}$ and an area of $1.35 \mathrm{mx} 0.8 \mathrm{~m}(1.08 \mathrm{~m} 2)$. Tanks to collect the distilled water were placed on the four walls of the system, a mirror at the back as a reflector; and temperature sensors were also placed for thermal characterization. During a day with a volume of 25 liters of salt water in the tank, 2.19 liters of distilled water were obtained, in the glass cover 1.12 liters $(51.12 \%)$, left side wall 0.52 liters of $(23.75 \%)$ and 0.55 liters of the right side wall $(25.23 \%)$, the insulated reflective wall did not condense water vapor; the salt concentration of the seawater from an initial value of 41.3 was lowered to 0.004 grams of salt/liter of the water, while the $\mathrm{pH}$ of 7.87 and 7.03. The distillation efficiency on the vertical side walls of glass was $3.051 / \mathrm{m}^{2}$ day was greater than the $30^{\circ}$ inclined cover of glass equal to $0.751 / \mathrm{m}^{2}$ day, approximately four times compared to the $30^{\circ}$ inclined cover.

The results indicated that the DSV is a good alternative to distill seawater at small volumes per day, at low cost for human consumption and other uses, in the case of requiring larger volumes of distilled water, the systems can be used in a modular way.
\end{abstract}

Keywords: Water, still, solar, reflectingsurface, temperature, volume.

\footnotetext{
${ }^{1}$ Universidad Nacional Jorge Basadre Grohmann, Facultad de Ciencias. Tacna, Perú. E-mail: cpolob@ unjbg.edu.pe

${ }^{2}$ Universidad Nacional Jorge Basadre Grohmann, Facultad de Ciencias. Tacna, Perú. E-mail: aglas6@gmail.com
} 


\section{INTRODUCCIÓN}

La región de Tacna se caracteriza por su aridez y déficit hídrico, que cada año se acentúa y limita su desarrollo integral, equivalente a $11 \mathrm{~m}^{3} / \mathrm{s}$, razón que motivó la presente investigación consistente en destilar el agua de mar, usando la energía solar como una alternativa para obtener agua dulce para consumo humano a pequeña escala, en zonas cercanas al litoral regional, a lo indicado se suma que en la zona altoandina en las cabeceras de cuencas hídricas se tienen agua contaminada naturalmente por su alto contenido de Boro, Arsénico y otros metales que afectan la salud poblacional y a la agricultura regional, por encima de $0,01 \mathrm{mg}$ As/litro establecido en la Guía para la Calidad del Agua Potable de la Organización Mundial de la Salud y ratificado por el Perú en el Reglamento de la calidad de Agua para Consumo Humano (DIRESA, 2011).

En el Perú, los problemas de la gestión del agua son muy complejos y se acentúan por la falta de tecnologías bien establecidas y estudiadas para la desinfección y descontaminación del agua, toda vez que las metodologías tradicionales de tratamiento son extremadamente caras, por lo que, es necesario el desarrollo de tecnologías simples, eficientes, de bajo costo, no contaminantes para la remoción de minerales de las aguas, desinfección de elementos patógenos y microbianos y para la desalación de agua de mar, usando como fuente energética la energía solar y otras renovables (Litter M. et al, 2010).

Por otro lado, la región Tacna cuenta con uno de los mejores potenciales energéticos solares de nuestro país, estudios realizados en el CERT indican que el potencial promedio anual de la irradiancia solar global incidente sobre una superficie horizontal es de $6,01 \mathrm{KWh} / \mathrm{m}^{2}$ día, equivalente a 0,51 litros de petróleo por metro cuadrado de superficie del desierto en un día, encima del promedio mundial en un 13,46 \% (Polo, 2012), indicador que la convierte en muy atractiva para cualquier aplicación solar a pequeña y gran escala; y sobre todo un indicador energético fundamental para que la región pueda mejorar la oferta hídrica a través de la desalinización de agua de mar, usando la energía solar como fuente energética.

Actualmente, existen muchas técnicas para la destilación y desalación de agua de mar, basado en procesos térmicos, mecánicos y químicos, como por ejemplo, la destilación Multietapa, destilación Flash en vacío, Ósmosis Inversa y otros. La más difundida a nivel mundial es la Ósmosis Inversa (OI), las técnicas mencionadas requieren de gran cantidad de energía para su funcionamiento, y mano de obra calificada para su operación y mantenimiento, además de sus altos costos de instalación y producción de agua para consumo humano y agropecuario.

Prakash \& Natarajan (2015) indican que, adicionando en la cubeta del destilador bobinas helicoidales de cobre, aletas de aluminio, tubos huecos de acero inoxidable y planchas de hierro,se aumenta la productividad de agua destilada durante el día y las noches, reduciendo el tiempo de precalentamiento del agua en la cubeta.

Colmenares y Pinzón (2008) indican que, bajo el análisis teórico de materiales a usarse en la construcción y las características ambientales de la zona, es posible usar el destilador solar como una alternativa para obtener agua potable. Nandwani \& Marin (1986) concluyen que con el destilador de cobertor de vidrio presenta mayor rendimiento que el destilador con cobertor de plástico, que la cantidad de agua destilada varía entre 2 a 4 litros $/ \mathrm{m}^{2}$ día y que dentro del destilador hay una pérdida de agua del 25 al $60 \%$ y que el enfriamiento artificial del vidrio cobertor con agua, acelera el proceso de condensación. 
Torchia et al. (2009) afirman que la relación energía/exergía muestra que el componente más eficiente en el destilador solar es la masa de agua salada con más de $90 \%$ para cualquier valor de los parámetros estudiados mientras que el colector alcanza el $23 \%$ para un valor de 1000 $\mathrm{W} / \mathrm{m}^{2}$ de irradiancia solar. Esteban et al. (2002), establecen que las producciones y eficiencias del Destilador Colector Solar Acumulador (DCSA) respecto a uno con batea y otro con tipo batea con un colector plano, muestran que el DCSA tiene una producción de un $70 \%$ más que el tipo batea y de un $20 \%$ que el tipo batea con colector plano.

Polo y Mamani (2012), con un destilador solar tubular para desalinización de agua de mar de $110 \mathrm{~cm}$ de largo, $4 \mathrm{~cm}$ de diámetro interno, bajo las condiciones meteorológicas de la ciudad de Tacna, indican que, han obtenido agua destilada para días soleados en valor promedio 2950 $\mathrm{ml} / \mathrm{m}^{2}$ día, en días semi nublados $1600 \mathrm{ml} / \mathrm{m}^{2}$ día y en días nublados $200 \mathrm{ml} / \mathrm{m}^{2}$ día; con eficiencias promedios de 62, 47 y $18 \%$, respectivamente; en tanto, Ruiz (2002) indica que con un destilador de una vertiente de $40 \mathrm{mx} 1,60 \mathrm{~m}$ la producción diaria fue de 1,6 $\mathrm{m}^{3}$, las cubiertas de vidrio se instalaron a un ángulo mayor de $45^{\circ}$, se observó que la producción de estas unidades fue mayor que la de las cubiertas con cerca de $15^{\circ}$ de pendiente.

El uso de los destiladores solares en la actualidad no es muy difundido en nuestra región debido a la escasa o nula información que existe en nuestro país, ya sea por la falta de equipos apropiados, limitaciones económicas o el poco interés comercial que este tiene a nivel nacional y/o local. La construcción del Destilador Solar de una Vertiente (DSV) se ha realizado a partir de un modelo ya existente desarrollado por Shyam S. Nandwani, al cual, se le realizó algunas modificaciones como el espejo reflector, paredes laterales verticales de vidrio. En este marco, se desarrolla la investigación, que tiene como objetivo construir y caracterizar térmicamente un destilador solar de una vertiente para desalar agua de mar (DSV), bajo las condiciones meteorológicas de la ciudad de Tacna, utilizando la energía solar como fuente energética y desarrollar el "now how" en la construcción y la evaluación de destiladores solares.

\section{Destilador solar de una vertiente con superficie reflejante (DSV)}

La radiación solar incidente sobre el vidrio cobertor y la reflejada por el espejo de la pared posterior, se transmiten a través del vidrio y son absorvidas por el fondo metálico de la cubeta pintado de negro y el agua contenida, con el aumento de temperatura, se produce la evaporación del agua en todo el volumen de la cámara del destilador aumentando la humedad relativa interior, que se condensa al entrar en contacto con la cubierta de vidrio inclinada y paredes laterales, que se encuentra a menor temperatura debido a que está en contacto con el aire del ambiente que lo rodea, el que actúa como medio refrigerante en permanente contacto con el vidrio (fenómeno de rocío). El agua condensada se desliza por gravedad por el plano inclinado del cobertor de vidrio y paredes laterales verticales hasta llegar a los tubos recolectores. En este proceso, se transfiere energía calorífica desde la cubeta con agua hacia la cubierta de vidrio por convección, radiación y por el proceso de evaporación condensación. A su vez, hay pérdidas de calor hacia el medio ambiente, principalmente a través del vidrio transparente por convección y radiación y por conducción por el fondo y paredes laterales del destilador (Donald, 1999). 


\section{Figura 1}

Vista general del destilador de una vertiente, mostrando el vidrio cobertor, pared posterior con espejo de vidrio y sistema de recolección del agua destilada

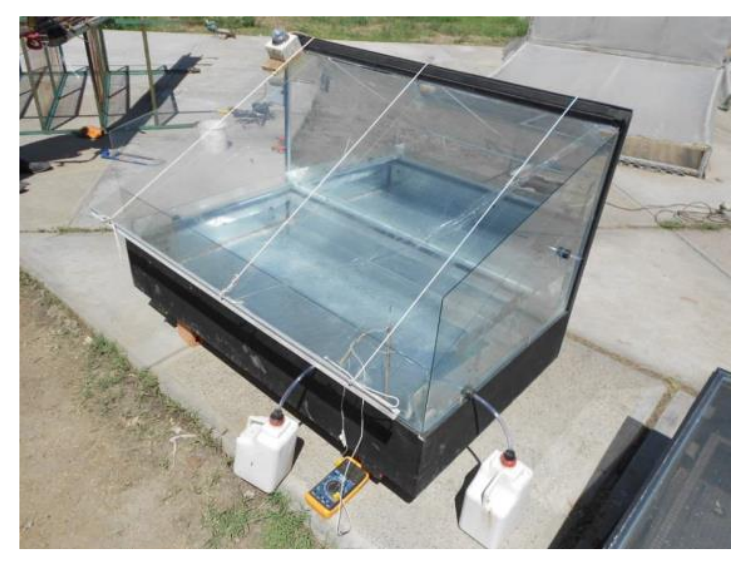

\section{Pérdidas de energía en el destilador solar}

En el esquema del DSV de la Figura 2, se muestra el balance energético en funcionamiento para destillar agua de mar, usando como fuente energética la energía solar. Transferencia de calor por evaporación-convección desde el agua de mar depositada en la bandeja hacia aire húmedo en el interior de DSV; transferencia de calor por convección-condensación ocurre desde aire húmedo interno hacia la cubierta interna y transferencia de calor por radiación entre el agua superficial y cubierta interna de vidrio; radiación-convección desde la cubierta exterior de vidrio hacia la atmósfera. La bandeja negra de fierro galvanizado transfiere calor por convección al estar en contacto por el lado superior con el aire húmedo y agua de mar, en el interior del DSV. El agua de mar es evaporada y transferida al aire húmedo y finalmente es condensado en la superficie interna de la cubierta de vidrio (Duffie \& Beckman, 2013), (Holman, 1999).

Donde:

It $\quad$ irradiancia solar incidente sobre el plano del cobertor de vidrio $\left(\mathrm{W} / \mathrm{m}^{2}\right)$, es la suma de la irradiancia solar directa (Ib) y la difusa (Id)

Qc = calor perdido por conducción por el fondo, $\mathrm{y}$ laterales del DSV

Qca = calor absorbido por el agua de la cubeta

QN = calor que ingresa al interior del DSV

Qrw = calor emitido por el agua y el fondo negro de la cubeta

Qe = calor utilizado para la evaporación

Qcw = calor utilizado para la convección que transporta las moléculas de agua evaporadas hacia el interior del vidrio cobertor 
Figura 2

Diagrama esquemático de las pérdidas de calor desde un destilador Solar a una pendiente (DSV)

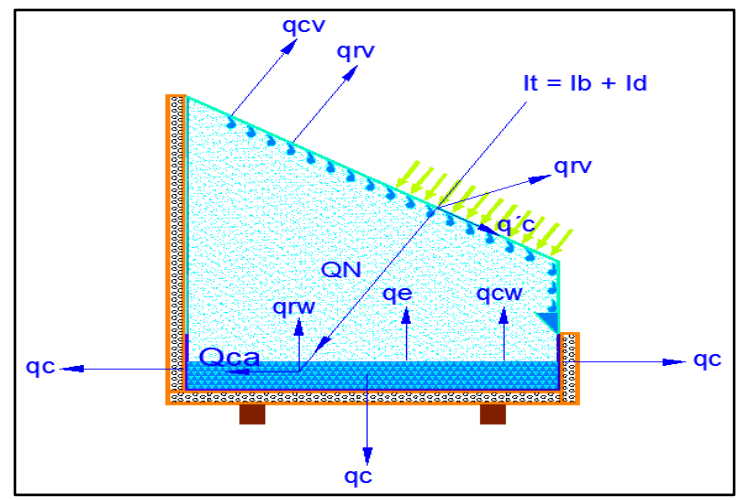

Qrve = irradiancia solar reflejada por el vidrio cobertor hacia el medio ambiente

$\mathrm{Qcv}=$ calor perdido por conducción-radiación a través del vidrio cobertor

Qrv = calor perdido por radiación-convección desde el vidrio cobertor

$\mathrm{Q}^{\prime} \mathrm{c}=$ calor perdido por la condensación del agua evaporada en el interior del cobertor

Del cual establecemos la siguiente ecuación:

$\mathrm{It}=\mathrm{QN}+\mathrm{Qca}+\mathrm{qrw}+\mathrm{qe}+\mathrm{qcv}$

$-\mathrm{qc}-$

qrve $-q c v-q r v-q^{\prime} c$

\section{MATERIAL Y MÉTODOS}

Los materiales utilizados en la construcción del DSV son 2,96 $\mathrm{m}^{2}$ de vidrio transparente de 3 $\mathrm{mm}$ de espesor; $1,08 \mathrm{~m}^{2}$ de espejo de vidrio; $6,46 \mathrm{~m}^{2}$ de planchas de tripley de $6 \mathrm{mmm}$ de espesor; $1,89 \mathrm{~m}^{2}$ de plancha de latón galvanizado de 1/32" de espesor; $1,02 \mathrm{~m}^{2}$ de plancha de tecnopor de 1" de espesor, pintura negra mate, $2 \mathrm{~m}$ de manguera de $1 / 4$ ", un tubo de silicona, $1 / 2$ Kg de clavos de 1/4", y $1 / 4 \mathrm{Kg}$ de 1/2".

En la construcción se utilizaron materiales locales. Las dimensiones internas del destilador son de $0,80 \mathrm{~m}$ de altura en la parte trasera, $0,75 \mathrm{~m}$ de ancho, $1,35 \mathrm{~m}$ de largo y 0,35 $\mathrm{m}$ de altura en la parte frontal, con una cámara de $0,58 \mathrm{~m}^{3}$, con un espesor de $3 \mathrm{~cm}$, el cobertor tiene una inclinación de $30^{\circ}$ y área de $1,35 \mathrm{mx} 0,8 \mathrm{~m}\left(1,08 \mathrm{~m}^{2}\right)$, para colectar el agua destilada, en las cuatro paredes del sistema se colocaron tubos y depósitos recolectores, la pared trasera es reflectante con espejo de vidrio.

Para la evaluación experimental del destilador solar, se usó un sistema de adquisición de datos marca Squirrel SQ 1200 Series, una estación meteorológica Vantage Pro Weather Station (Davis Instruments) ubicada en la cima del tercer piso del pabellón de estudios de la Facultad de Ciencias, frente al CERT, un Piranómetro Moll-Gorczynsky marca Kipp and Zonen, de sensibilidad espectral comprendido de 0,3 a 3 um con un tiempo de respuesta de 5 segundos, siete termopares de Cromel-Alumel, como sensores de temperatura, ubicadas en diferentes 
partes del destilador solar, un cronometro digital, un multímetro digital, probeta milimetrada, tal como se detalla en la Figura 3.

\section{Figura 3}

Ubicación de los sensores para la evaluación y caracterización del DSV (3A)

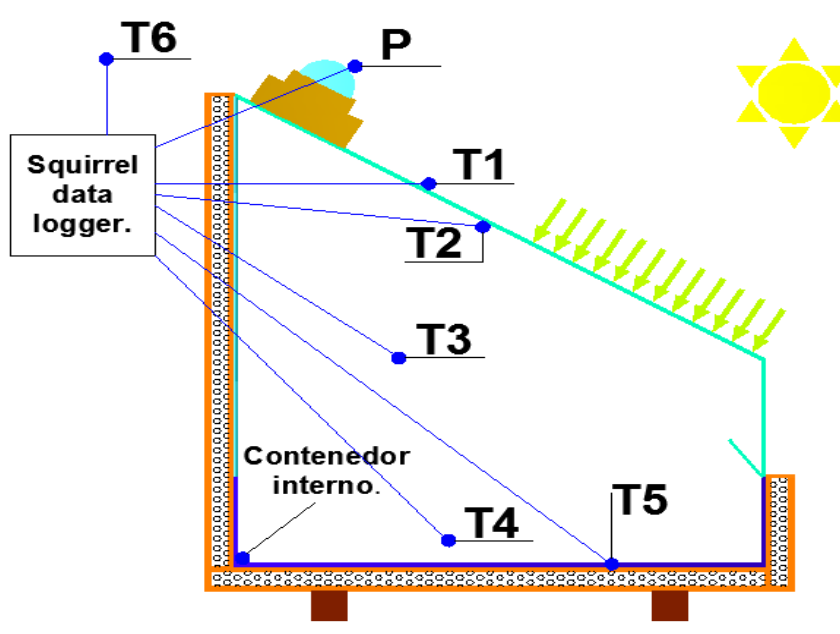

Nota:

T1: temperatura de la superficie externa del vidrio, T2: temperatura de la superficie interna del vidrio, T3: temperatura del vapor en el interior del DSV, T4: temperatura del agua almacenada en la cubeta, T5: temperatura de la superficie del fondo de la cubeta, T6: temperatura ambiente, P: irradiación global incidente al DSV medido por el piranómetro.

Las temperaturas en el sistema e irradiancia solar sobre el plano del cobertor se registran continuamente cada 15 segundos, en tanto que los volúmenes del agua destilada en superficie lateral izquierda V2, en superficie lateral derecha V3, en cobertor inclinado V1 y en superficie reflejante posterior V4 a efectos de incrementar la irradiancia solar incidente sobre la superficie de la cubeta metálica, se almacenan en depósitos de plástico, para luego cuantificarlos cada hora con una probeta graduada en mililitros (Figura 4).

\section{Figura 4}

Ubicación de los depósitos para colectar el agua destilada de las cuatro paredes internas en el DSV

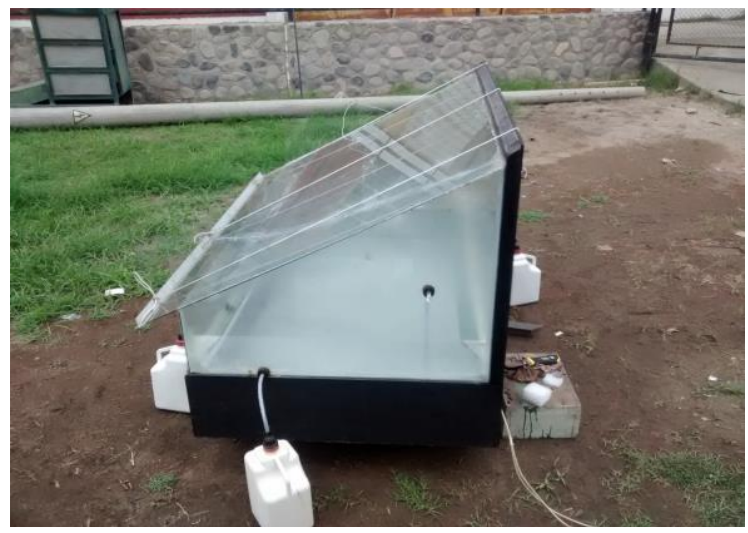

\section{RESULTADOS Y DISCUSIÓN}




\section{Evaluación del DSV en vacío}

En un día, con un volumen de 15 litros de agua salada en la cubeta, se obtuvo 2,19 litros de agua destilada, se cuantificó el volumen de agua destilada obtenida en cada una de sus partes del destilador es diferente, así en el cobertor inclinado de vidrio fue de 1,12 litros (51,12\%), 0,52 litros en la pared lateral izquierda $(23,75 \%)$ y 0,55 litros en la pared lateral derecha $(25,23$ $\%)$, la pared reflectante no condensa vapor de agua.

En la Figura 5, se muestra la variación de temperatura en los diferentes puntos de monitoreo para funcionamiento del destilador sin agua en la cubeta (en vacío) durante un día completo entre las 07:00 a 17:30 horas, el fondo del recipiente alcanzó $105^{\circ} \mathrm{C}$ (T5), mostrando que la superficie metálica es buena receptora de la radiación solar incidente; en tanto que la ambiental alcanza $26^{\circ} \mathrm{C}$ (T6), el gradiente de temperatura existente entre las superficies interna y externa del vidrio varía entre $\left(2\right.$ a 7) ${ }^{\circ} \mathrm{C}$ durante el día alcanzando valores máximos entre $47{ }^{\circ} \mathrm{C}(\mathrm{T} 1)$ y $51{ }^{\circ} \mathrm{C}(\mathrm{T} 2)$, respectivamente; en tanto que la temperatura del aire interno cerca del fondo (T4) es ligeramente mayor que la del centro de la cámara (T3), con valores máximos de $90{ }^{\circ} \mathrm{C}$ y 85 ${ }^{\circ} \mathrm{C}$, respectivamente, no son uniformes; los valores indicados son bajo irradiancia solar incidente instantánea de $914 \mathrm{~W} / \mathrm{m}^{2}$ (Figura 5).

\section{Figura 5}

Variación de temperaturas en el destilador solar de una vertiente evaluado en vacío en función de las horas del día

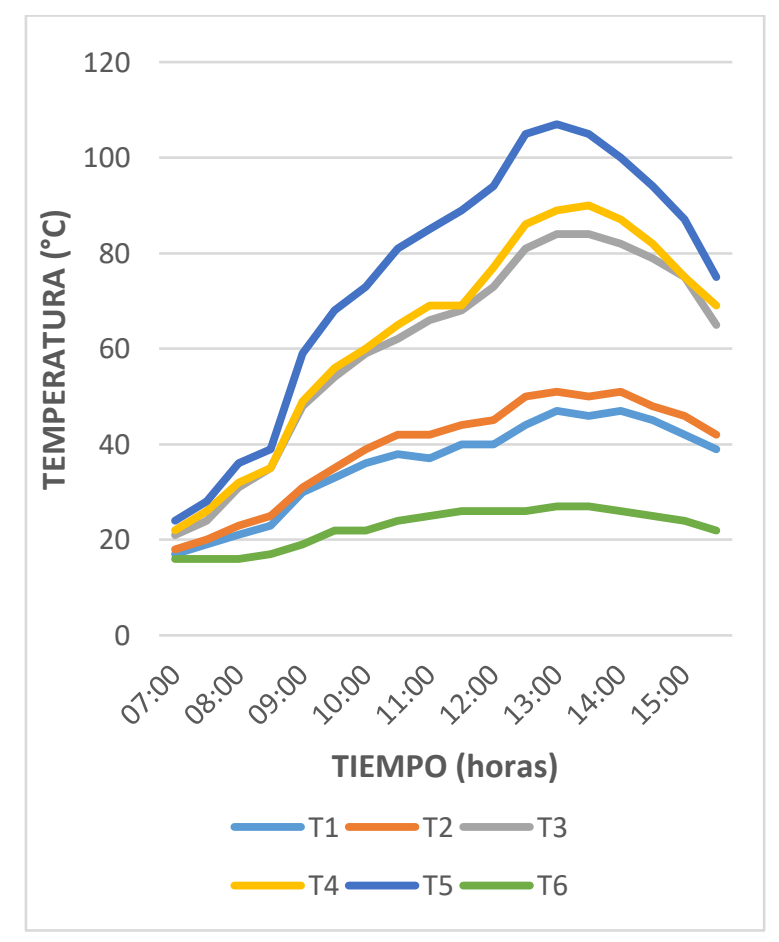

En la Figura 5, puede apreciarse que todas las temperaturas disminuyen con referencia a la Figura 4, a excepción de T3, debido a la masa térmica del vapor de agua.

Se observa que T3, se eleva por encima de todas, lo cual confirma que el vapor de agua en el medio interno del DSV existe un gradiente vertical negativo de temperatura, mucho mayor antes de hacer contacto con la superficie interna del vidrio. Es este cambio brusco de temperatura que contribuye a la condensación del vapor de agua. 


\section{Evaluación del DSV bajo funcionamiento}

Para el efecto, se depositó en la bandeja metálica de $1,013 \mathrm{~m}^{2}$ de superficie de fondo un volumen de agua de $0,025 \mathrm{~m}^{3}$ con una temperatura inicial de $18{ }^{\circ} \mathrm{C}$ que representa un nivel aproximado de 2,5 cm de altura en dicha bandeja. El agua destilada o condensada es colectada en las cuatro superficies de la cámara del destilador (tres laterales y cobertor inclinado) y almacenada en los contenedores externos (V1, V2, V3, V4), obteniendo en un día de evaluación, $230 \mathrm{ml}$ en V1, $1580 \mathrm{ml}$ en V2 y $200 \mathrm{ml}$ en V3, y V4 cero $\mathrm{ml}$. Se encontró fugas por goteo en el vidrio inclinado, fugas de vapor en orificios no sellados y además, se observó que la evaporación-condensación del agua se inicia pasada las tres horas de exposición a la irradiancia solar. La variación de temperaturas en diferentes puntos del destilador se muestra en la Figura 6.

\section{Figura 6}

Variación de la temperatura en el destilador solar de una vertiente bajo destilación de agua en función de las horas del día

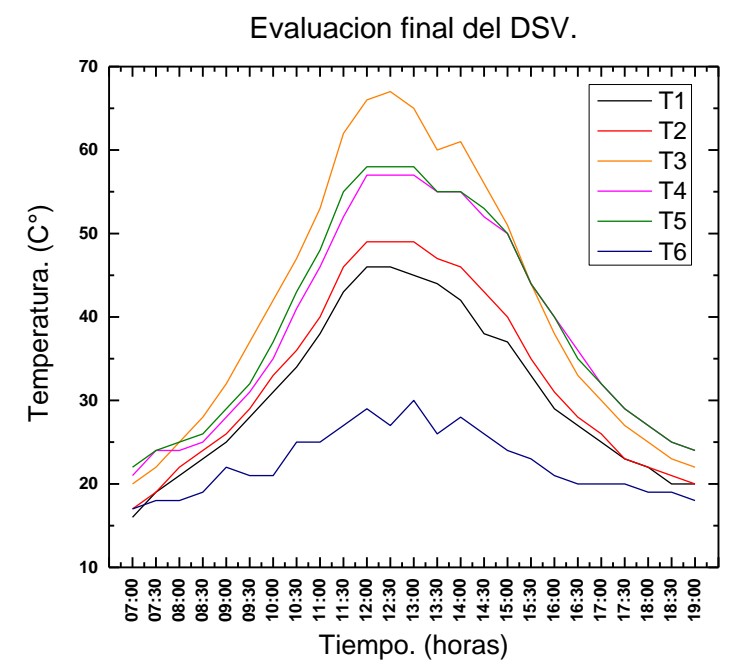

La temperatura siempre es mayor en el fondo de la cubeta metálica, alcanzando valor máximo de $68^{\circ} \mathrm{C}$.

En la Figura 7, se puede apreciar que no se produce destilación alguna durante aproximadamente tres horas después de iniciado el funcionamiento, lo cual, se debe a que el destilador se carga con 25 litros de agua a temperatura de la red, por lo que existe un tiempo de precalentamiento del agua en la cubeta de tres horas que afecta la producción de agua destilada, en concordancia con lo establecido por Prakash y Natarajan (2015). Alcanza su máxima producción entre las 13 y 14 horas del día cuando la irradiancia solar sobre el plano del cobertor es de $900 \mathrm{~W} / \mathrm{m}^{2}$. 


\section{Figura 7}

Volumen de agua destilada por día e irradiancia solar incidente sobre el plano de cobertor inclinado de vidrio del DSV en función de las horas del día.

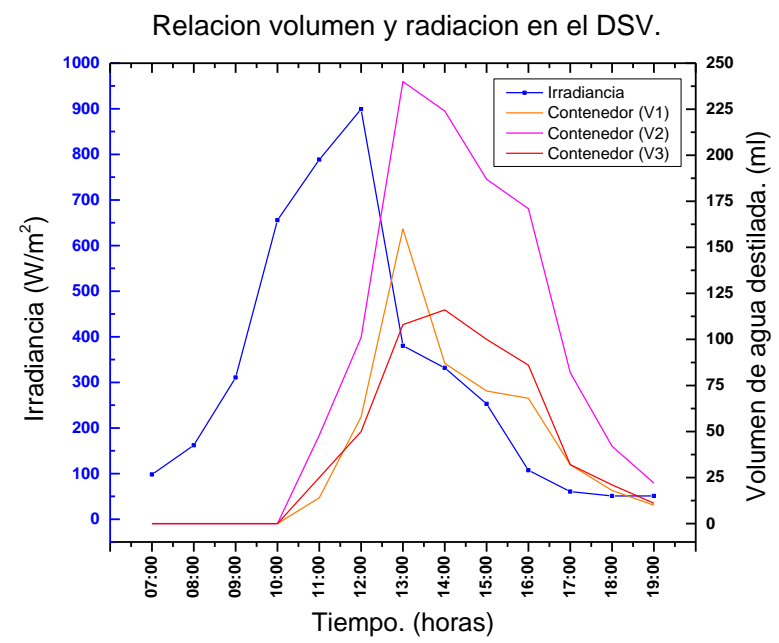

Además, puede observarse que a medida que incrementa la irradiación, incrementa el volumen de agua destilada, determinando así la influencia de la irradiancia en el DSV para producir agua destilada. También se puede verificar la contribución de los vidrios laterales conectados a los colectores V1 y V3 cuya ubicación de los mismos se muestra la Figura 4.

En la Figura 8, se muestra el perfil diario de la producción de agua destilada en cada uno de los colectores de las paredes laterales verticales de vidrio y cobertor inclinado del destilador, cuantificados cada hora.

\section{Figura 8}

Volumen de agua destilada por el Destilador Solar de una Vertiente (DSV) en cada pared lateral y el cobertor en función de las horas del día.

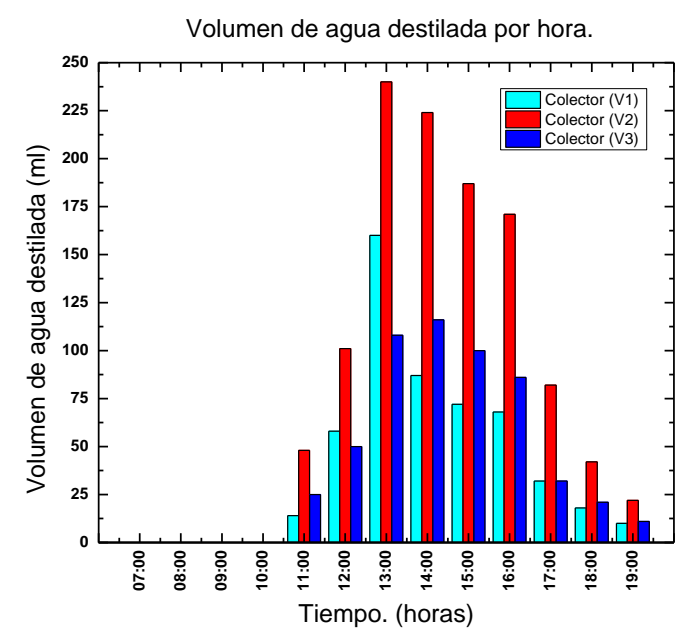

El volumen promedio de agua destilada por día en el depósito V2 correspondiente al condensado por la pared vertical derecha es de $519 \mathrm{ml}$, para el cobertor inclinado $30^{\circ}$ de vidrio 
V1 de $1117 \mathrm{ml}$ y para el de pared vertical derecha V3 de $549 \mathrm{ml}$, sumando un total de $2185 \mathrm{ml}$. La superficie reflejante y asilada térmicamente no condensa una sola gota de vapor, debido a que la superficie interna está a la misma temperatura del vapor de agua no existiendo un gradiente térmico que haga posible la condensación del vapor de agua.

El rendimiento de destilación considerando el área de las paredes de vidrio verticales laterales y la del cobertor inclinado, en las paredes laterales es de 3,05 litros $/ \mathrm{m}^{2}$ día, en tanto que en el cobertor de 0,75 litros $/ \mathrm{m}^{2}$ día, lo que significa que en las paredes verticales la condensación es aproximadamente mayor en cuatro veces respecto al inclinado en $30^{\circ}$, producción mayor a cualquier destilador simple y similar a destiladores con materiales metálicos en la bandejas, según lo indicado por Pakash \& Natajaran (2015) y en concordancia con lo establecido por Nandwani \& Marin (1986). La producción diaria del volumen de agua destilada por las paredes verticales de vidrio es mayor a lo obtenido con un destilador solar tubular desarrollado por Polo C. \& Mamani E. (2012).

Por otro lado, se realizaron ensayos con un volumen inicial de 25 y 15 litros de agua en la cubeta, obteniendo mayor volumen de agua destilada con 15 litros de agua en la cubeta, por lo que existe una mayor eficiencia de destilación a menor volumen, a pesar de las condiciones adversas del clima.

\section{Concentración de sal y pH}

En laboratorio, bajo análisis físico-químico se determinó que, bajo el proceso de destilación solar del agua de mar, con una concentración inicial de 41,3 gsal/1 agua en un día se bajó a $0,004 \mathrm{~g} \mathrm{sal} / \mathrm{l}$ agua, en tanto que el $\mathrm{pH}$ de 7,87 a 7,03, estos valores nos indica que el agua es de buena calidad apta para el consumo humano y para utilizarla en cualquier proceso industrial.

\section{CONCLUSIONES}

El Destilador Solar de una Vertiente (DSV) muestra un desempeño razonable en la destilación de agua de mar, a razón de 2,185 litros/día en promedio, bajo las condiciones meteorológicas de la ciudad de Tacna, con irradiancia solar promedio de $165 \mathrm{~W} / \mathrm{m}^{2}$, temperatura ambienta variable entre $18^{\circ} \mathrm{C}$ y $23^{\circ} \mathrm{C}$, con 25 litros iniciales de agua de mar en la bandeja, con una superficie colectora de $1,013 \mathrm{~m}^{2}$, y volumen de cámara de aire húmedo de $0,58 \mathrm{~m}^{3}$.

El vapor de agua no solo se condensa en el vidrio cobertor, sino también en los vidrios de las paredes laterales verticales derecha e izquierda, más no en la pared posterior reflejante-aislada térmicamente debido a que no se establece un gradiente térmico entre la superficie interior y exterior.

El rendimiento de destilación es diferente en las paredes verticales laterales de vidrio y la del cobertor inclinado $30^{\circ}$ de vidrio, en las paredes laterales es de 3,05 litros $/ \mathrm{m}^{2}$ día mucho mayor que en el cobertor de 0,75 litros $/ \mathrm{m}^{2}$ día, lo que significa que en paredes verticales la condensación es aproximadamente mayor en cuatro veces respecto al cobertor inclinado en $30^{\circ}$. El volumen de agua destilada por día, depende de la irradiancia solar global incidente, la temperatura inicial, el volumen de agua de mar depositado inicialmente en la cubeta y de la temperatura del agua a destilar; a menor volumen inicial de agua en la cubeta, mayor es el volumen de agua destilada por día.

Los destiladores de una vertiente pueden ser una alternativa para disponer de agua para consumo humano cuando se presentan emergencias por desastres naturales, como huaycos, 
inundaciones, terremotos, toda vez que el agua obtenida puede ser usada para el consumo poblacional en el litoral regional y en lugares donde se tenga aguas contaminadas, entre otros usos diversos.

Recomendamos realizar pruebas experimentales para ver la factibilidad de remover otros tipos de sales como el Boro o Arsénico, elementos que se encuentran presentes en el agua de todas las cuencas hídricas de la región Tacna y otras regiones de nuestro país.

\section{REFERENCIAS}

Colmenares, S. y Pinzón, J. (2008). Diseño, construcción y pruebas de un destilador solar. Universidad Pontificia Bolivariana. Colombia.

Dirección General de Salud Ambiental (DIGESA) (2011). Reglamento de la calidad de agua para consumo humano, DS Nº31-2010-SA. Ministerio de Salud. Lima, Perú.

Donald, Q. K. (1999). Procesos de transferencia de calor. Cecsa. Mexico

Duffie, J. A., \& Beckman, W. A. (2013). Solar Engineering of Thermal Processes. Madison: University of Wisconsin-Madison. USA

Esteban, C., Franco, J. y Fasulo, A. (2012). Evaluación experimental de un destilador colector solar acumulador. Avances de Energías Renovables y Medio Ambiente, 6(1).

Holman, J. (1999). Transferencia de calor. España: Mc Graw Hill.

Kalogirou, S. (2009). Solar energy engineering: processes and systems. Elsevier Inc. San Diego. USA

Litter, M., Sandia, A. y Ingallanela, A. (2010). Tecnologías Económicas para el abatimiento de arsénico en aguas. Cyted, Iberoarsen. Argentina.

Nandwani, S. \& Van Koller, M. (1986). Estudio preliminar de tres modelos de destiladores solares para producir sal y agua potable. UNICIENCIA 3 (1-2), 59 - 69.

Polo Bravo, C. (2013). Cuantificación del Potencial Energético Solar y su Impacto Ambiental en la región Tacna, tesis de Maestría, ESPG-UNJBG. Tacna, Perú.

Polo Bravo, C. y Mamani, F. (2012). Diseño, construcción y caracterización de un destilador solar tubular para desalinizar agua de mar. UNJBG, XIX Simposio Peruano de Energía Solar. Piura, Perú.

Prakash, M. \& Natarajan, E. (2015). Productivity enhancement of a single basin and single slope solar still coupled with variaus basin materials. Taylor y Francis. India.

Torchia, J. y Cervantes de Gortari, J. (2013). Discusión sobre el modelado de la destilación solar: Experimentos y Teoría. Tecnología en marcha, 26 (4), 00-108. 